\title{
Metais Poluentes em Sedimentos Fluviais de Cursos D'agua sob Influência de Pecuária Leiteira no Estado de Goiás, Brasil
}

\author{
Cleonice Rocha ${ }^{1}$, Alfredo Borges de Campos ${ }^{2}$, Francisco Leonardo Tejerina Garro ${ }^{3}$, \\ Affonso Celso Gonçalves Jr. ${ }^{4}$ \\ rcleonice@gmail.com; Alfredo.borges.campos@gmail.com; garro@pugoias.edu.br; affonso133@hotmail.com
}

Recebido: 09/07/13 - revisado: 15/07/13 - aceito: 29/08/13

\begin{abstract}
RESUMO
O nivel e fonte de contaminação de ecossistemas aquáticos são frequentemente determinados pela concentração de metais poluentes em seus sedimentos. Neste trabalho verificou-se a influência da atividade pecuária leiteira na contaminação de sedimentos por $\mathrm{Cu}, \mathrm{Fe}, \mathrm{Mn}, \mathrm{Zn}, \mathrm{Cd}, \mathrm{Pb}, \mathrm{Cr}$ e Al. As amostras foram coletadas a jusante e montante de aguadas utilizadas para dessedentação de gado em 31 cursos d'água da microrregião Meia Ponte, principal bacia leiteira do estado de Goiás. Os metais foram analisados por espectrometria de absorção atômica nas frações menores que 0,063 mm após digestão ácida. Com exceção do Al e Zn, os demais metais apresentaram baixas concentrações. A comparação com os valores guias canadense de qualidade de sedimentos (VGQS) mostrou que o Zn apresenta risco potencial de contaminação da biota, enquanto o $\mathrm{Cu}, \mathrm{Cd}, \mathrm{Pb}$ e $\mathrm{Cr}$ apresentam baixo risco. O Zn apresentou maior concentração a montante e os demais metais não apresentaram diferenças significativas nas concentrações obtidas a montante e a jusante. Conclui-se que os metais analisados são provenientes de processos geológicos e que a atividade pecuária leiteira na região não contribui para a contaminação dos recursos hidricos com estes metais.
\end{abstract}

Palavras-Chave: Metais. Contaminação. Atividade leiteira.

\section{INTRODUÇÃO}

Em países desenvolvidos ou em desenvolvimento a contaminação de ecossistemas aquáticos, e consequentemente de sedimentos a eles associados, está frequentemente relacionada ao crescimento populacional, urbanização, atividades agropecuárias e industriais (MUNIZ; OLIVEIRA-FILHO, 2006; OLADE, 1987).

O Brasil é um dos maiores produtores mundiais de leite, ficando no ranking internacional atrás apenas dos Estados Unidos, Índia e China (FAO, 2012). Dentre os estados brasileiros, Goiás é o quarto maior produtor de leite sendo a microrregião Meia Ponte a que apresenta maior produção no Estado (IBGE, 2011). Nessa microrregião é comum o sistema produtivo extensivo, no qual o gado leiteiro utiliza aguadas para dessedentação e deposita nestas grandes quantidades de fezes e urina enrique-

\footnotetext{
${ }^{1}$-Dep. Matemática e Física/PUC - Goiás

${ }^{2}$-Instituto de Geociências/UNICAMP

${ }^{3}$-Dep. Biologia/ PUC - Goiás

${ }^{4}$-Centro de Ciênc ias Agrárias/UNIOESTE - PR
}

cendo a água e sedimentos de fundo de cursos fluviais com matéria orgânica, nutrientes e demais componentes destes dejetos, como fósforo, nitrogênio, herbicidas, inseticidas e metais utilizados na manutenção de pastos e tratamento do animal (CEREJEIRA; VIANA; BATISTA, 2003; FITCH; ADAMS, 1998; LIM et al., 1998; MARÇAL et al., 2003; ORRICO JÚNIOR et al., 2012; SHIGAKI; SHAPPLEY; PROCHONOW, 2006). A adição contínua dos compostos presentes nas fezes e urinas pode ao longo do tempo causar a contaminação do meio aquático e sedimentos fluviais (DOWNEY; MOORE, 1977; TIMOFIECSYK et al., 2012).

Sedimentos podem apresentar níveis elevados de metais poluentes advindos de processos naturais ou antropogênicos (JESUS et al., 2004). Poluentes associados a processos naturais podem ser acumulados em sedimentos a partir da coluna d'água que mesmo com baixa concentração de metais pode, em longo prazo, aumentar a concentração destes nas partículas sedimentares em várias ordens de grandeza, maiores do que aquelas encontradas na água. Sedimentos fluviais acumulam metais por serem ricos em carbonatos, sulfetos, matéria orgânica, argilas e óxidos de ferro e manganês que servem como adsorventes de metais (EBRAHIMI; TAHERI- 
ANFARD, 2011; MORILLO; USERO, J.; GRACIA, 2004; TESSIER; CAMPBELL, 1987). O comportamento e biodisponibilidade de metais em águas naturais é uma função da composição dos sedimentos e das propriedades químicas da água, que devido a fenômenos de dissolução, precipitação, sorção e complexação (NOURI et al., 2011) podem sofrer diversas transformações em sua especiação (ABDELGHANI; ELCHAGHBY, 2007; AKCAY; OGUZ; KARAPIRE, 2003), tornando-se mais ou menos solúveis.

Por outro lado, os sedimentos além de serem considerados potenciais reservatórios de substâncias tóxicas (ADAMS; KIMERLE; BARNETT, 1992) consistem em habitat e fonte de nutrientes para organismos aquáticos, o que os torna importantes na avaliação da qualidade total do ecossistema de um corpo de água (ADEYEMO et al., 2008; DAVIES; ABOWEI, 2009).

Metais estão presentes naturalmente em plantas e animais e quando em baixas concentrações não são nocivos, mas se tornam tóxicos em concentrações elevadas (MORGANO et al., 2005; VRIES; ROMKENS; SCHUTZE, 2007), a exceção de chumbo, cádmio e mercúrio que são tóxicos mesmo em concentrações baixas (ALLOWAY, 1995). Assim, a contaminação de sedimentos fluviais por metais é de interesse devido sua influência nas águas superficiais, plantas, animais e seres humanos (SUCIU et al., 2008). O acúmulo de metais em sedimentos fluviais pode intoxicar organismos aquáticos e peixes, resultando em morte, redução no crescimento e diversidade de espécies e ainda afetar a capacidade reprodutiva da biota (EBRAHIMI; TAHERIANFARD, 2011; PRAVEENA et al., 2007).

Apesar da importância de metais em sedimentos fluviais, no Brasil ainda não foram estabelecidos valores de referência para teores de metais nesse tipo de sedimento. A única regulamentação que se aproxima do assunto é dada pelo CONAMA na Resolução 454 de 01 de novembro de 2012 que estabelece as diretrizes e os procedimentos mínimos para dragagem de materiais em águas brasileiras (BRASIL, 2012). Esta resolução é baseada em valores guias de qualidade de sedimentos (VGQS) estabelecidos pelo Conselho Canadense do Ministério do Meio Ambiente (CCME).

Os VQGS baseiam-se em valores das concentrações totais de substâncias em sedimentos coletados em superfície (até $5 \mathrm{~cm}$ de espessura). A partir de informações químicas e toxicológicas presentes em um banco de dados do CCME, foram definidos dois valores limites para cada substância: o TEL (Threshold Effect Level: concentração de efeito limiar) e o PEL (Probable Effect Level: concentração de efeito prová- vel) (CCME, 1999a). O menor valor limite (TEL) representa o limite superior da concentração onde não foram encontrados efeitos adversos para os organismos aquáticos, enquanto o maior valor limite (PEL) representa o limite inferior de concentração associado a efeitos nocivos à biota (CCME, 1999a).

Assim, o objetivo deste estudo foi verificar a influência da atividade pecuária leiteira na contaminação de sedimentos fluviais coletados em cursos d'água localizados na microrregião Meia Ponte, estado de Goiás, pelos metais poluentes $\mathrm{Cu}, \mathrm{Fe}, \mathrm{Mn}$, $\mathrm{Zn}, \mathrm{Cd}, \mathrm{Pb}, \mathrm{Cr}$ e $\mathrm{Al}$. Na pesquisa buscou-se responder às seguintes perguntas: i) as concentrações dos metais nos sedimentos fluviais avaliados oferece risco a biota?; ii) as concentrações dos metais avaliados nos sedimentos são semelhantes aos níveis mundiais e nacionais?; iii) as concentrações de metais tóxicos nos sedimentos dos cursos fluviais avaliados são iguais entre si quando considerada a área geográfica ou a bacia hidrográfica amostrada?; iv) o uso de cursos d'água para dessedentação do gado leiteiro influencia na concentração dos metais tóxicos em sedimentos fluviais?

\section{MATERIAL E MÉTODOS}

\section{Área de estudo}

O estudo foi realizado na microrregião Meia Ponte em área localizada na região sudeste do estado de Goiás, delimitada a oeste pelo rio Meia Ponte, a leste pelo rio Piracanjuba e ao sul pelo rio Paranaíba (Figura 1). Nesta área a atividade predominante é a agropecuária e nela estão localizados os municípios de Morrinhos, Piracanjuba e Goiatuba que ocupam o $2^{\circ}, 3^{\circ}$ e $20^{\circ}$ lugar, respectivamente, na produção de leite em Goiás (IMB, 2012).

A pecuária leiteira utiliza o sistema de criação extensiva, o que implica na maioria dos casos na dessedentação do gado a partir de determinados pontos de acesso presentes ao longo dos cursos de água (aguadas), cuja localização fica evidenciada pelas trilhas de gado que são visíveis nas margens dos cursos d'água.

\section{Protocolos amostrais e análise dos dados}

Amostras de sedimentos fluviais foram coletadas nos primeiros $5 \mathrm{~cm}$ de espessura da coluna de sedimentos de fundo durante o período da estiagem (março a setembro) de 2007 em 31 cursos d'água de primeira ou segunda ordem hidrológica, que corresponde à designação comum de córregos ou ria- 


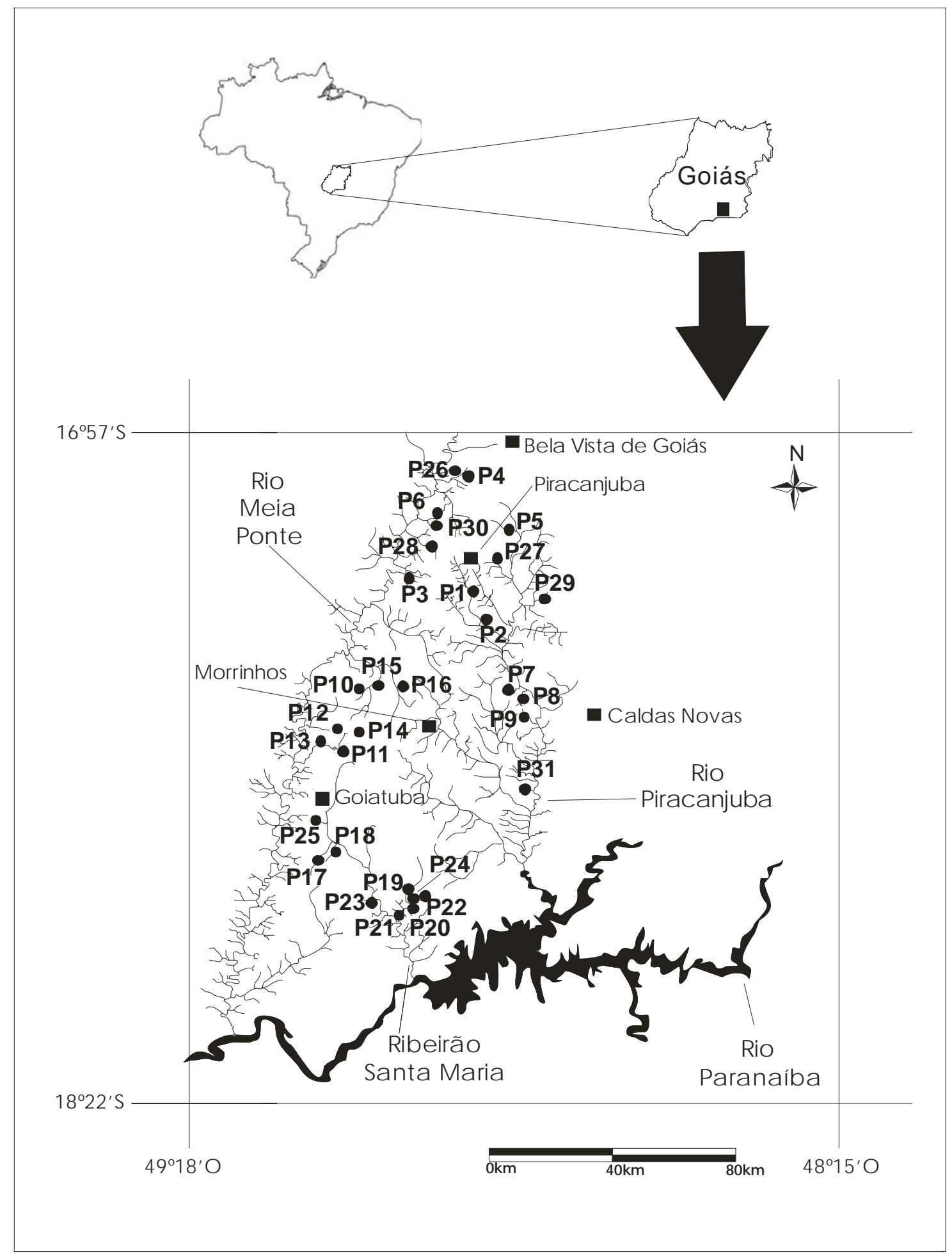

Figura 1 - Localização dos cursos d'água (P1 a P31) amostrados em 2007 nas bacias dos rios Meia Ponte, Piracanjuba e ribeirão Santa Maria pertencentes a bacia hidrográfica do rio Paranaíba em Goiás, Brasil Central. Quadrados pretos=cidades; Área preta no rio Paranaíba=Represa da Usina Hidrelétrica de Itumbiara 
chos. As coletas foram realizadas no período de estiagem, pois a concentração de metais em sedimentos pode ser influenciada pelo índice de chuvas que o curso d'água recebe (OLUBUNMI; OLORUNSOLA, 2010). Exceto os cursos d'água P11, P12 e P14, todos os outros cursos d'água amostrados apresentavam uma aguada, a partir da qual foram retiradas amostras de sedimentos fluviais a $50 \mathrm{~m}$ de distância a montante e a jusante do ponto da aguada. O sedimento foi coletado utilizando um amostrador Kajack provido de um tubo graduado de acrílico, e após coleta foi colocado num saco plástico e conservado numa caixa de gelo.

Em laboratório as amostras de sedimentos foram secas ao ar, peneiradas para obtenção de partículas < 0,063 mm (SANTOS; MELO JÚNIOR ; SEGUNDO, 2002) e digeridas com ácido nítrico e peróxido de hidrogênio de acordo com o método 3050B da USEPA (1995). O material digerido foi estocado em frasco de polietileno previamente lavado com ácido nítrico e enviado para o laboratório de Química Agrícola e Ambiental da Universidade do Oeste do Paraná (UNIOESTE-PR), onde foi feita a determinação da concentração dos metais alumínio $(\mathrm{Al})$, cádmio $(\mathrm{Cd})$, cromo $(\mathrm{Cr})$, cobre $(\mathrm{Cu})$, ferro $(\mathrm{Fe})$, manganês $(\mathrm{Mn})$, chumbo $(\mathrm{Pb})$ e zinco (Zn) pelo método de espectrometria de absorção atômica modalidade chama (EAA-chama).

Considerando que a Resolução CONAMA 454/2012 baseia-se nos VGQS canadense, neste trabalho também serão adotados os valores estabelecidos pelo Canadá para fins de comparação dos resultados.

A comparação dos resultados em nível mundial e nacional foi realizada por meio de revisão literária.

Os dados referentes às concentrações por curso d'água amostrado foram organizados numa matriz de análises e transformados $(\log x+1)$ para atingir os pressupostos de normalidade e homogeneidade. A partir desta matriz foi realizada separadamente duas ANOVA one-way, a primeira para comparar entre si as concentrações dos metais amostrados e a segunda para averiguar se houve diferenças entre as concentrações dos metais avaliados considerando o fator bacia hidrográfica (rios Meia Ponte e Piracanjuba, ribeirão Santa Maria). Ambas as análises foram seguidas de um teste post-hoc de Tukey. Finalmente foi realizado um teste de "t" para amostras dependentes a fim de averiguar os efeitos do uso das aguadas pelo gado nas concentrações de metais tóxicos no sedimento amostrado a montante e a jusante das mesmas.

\section{RESULTADOS E DISCUSSÃO}

Todos os metais analisados, com exceção do $\mathrm{Al}$ e $\mathrm{Zn}$, apresentaram concentrações baixas tanto a montante como a jusante das aguadas (Tabela 1).

Tabela 1 - Média da concentração $\left(\mathrm{mg} \cdot \mathrm{kg}^{-1}\right)$ dos metais analisados em amostras de sedimentos fluviais coletados a montante e a jusante dos pontos de aguada e valores de VQGS (mg.kg-1)

\begin{tabular}{|c|c|c|c|c|}
\hline Metal & Posição & Média $(\bar{x})$ & $\begin{array}{c}\text { VQGS } \\
\text { (TEL) }\end{array}$ & $\begin{array}{l}\text { VQGS } \\
\text { (PEL) }\end{array}$ \\
\hline \multirow[t]{2}{*}{$\mathrm{Al}$} & Montante & 220,08 & - & - \\
\hline & Jusante & 211,52 & & \\
\hline \multirow[t]{2}{*}{ Cd } & Montante & 0,0374 & 0,6 & 3.5 \\
\hline & Jusante & 0,0394 & & \\
\hline \multirow[t]{2}{*}{$\mathrm{Cu}$} & Montante & 3,2800 & 36,7 & 97 \\
\hline & Jusante & 2,8430 & & \\
\hline \multirow[t]{2}{*}{$\mathrm{Cr}$} & Montante & 0,8930 & 37.3 & 90 \\
\hline & Jusante & 0,8860 & & \\
\hline \multirow[t]{2}{*}{$\mathrm{Fe}$} & Montante & 2,0440 & - & - \\
\hline & Jusante & 1,7900 & & \\
\hline \multirow[t]{2}{*}{$\mathrm{Mn}$} & Montante & 3,5600 & - & - \\
\hline & Jusante & 3,1350 & & \\
\hline \multirow[t]{2}{*}{$\mathrm{Pb}$} & Montante & 0,5530 & 36 & 91.3 \\
\hline & Jusante & 0,5530 & & \\
\hline \multirow[t]{2}{*}{$\mathrm{Zn}$} & Montante & 181,27 & 123 & 315 \\
\hline & Jusante & 179,93 & & \\
\hline
\end{tabular}

Teores altos de $\mathrm{Al}$ são esperados uma vez que os Latossolos são os solos dominantes nas bacias hidrográficas pesquisadas e constituem área fonte dos sedimentos amostrados. Esses solos apresentam naturalmente elevadas concentrações desse metal em ambiente de Cerrado (MARQUES et al., 2004). Dessa forma, apesar de sua alta concentração não se pode dizer que os sedimentos analisados estão contaminados por Al.

Por outro lado, quando se compara os valores encontrados para o Zn com os VQGS estabelecidos pelo CCME (1999a) do Canadá, verifica-se que a concentração desse metal encontra-se entre os valores estabelecidos para o TEL e o PEL (Tabela 1). Neste caso, segundo preconiza o protocolo do VQGS, há risco potencial de contaminação da biota por Zn. Ressalta-se que o Zn tem alta afinidade por partículas presentes em meio aquático como óxidos de ferro e manganês e também pela matéria orgânica, e sua associação com esses materiais resulta na incorporação desse metal às partículas de sedimentos (CAMPBELL; TESSIER, 1996). Elevadas concen- 
trações de Zn nos sedimentos podem aumentar a mortalidade, alterar o comportamento e diminuir a diversidade e abundância de invertebrados bentônicos (CCME, 1999b). Dessa forma, há necessidade de futuras investigações para se determinar o quanto significante é o efeito nocivo do $\mathrm{Zn}$ associado aos sedimentos para os organismos aquáticos que vivem nos cursos d'água amostrados. Tais investigações devem incluir a determinação da concentração natural (valor referência) da substância que depende de pesquisas sobre a toxicidade do metal para as espécies presentes na biota aquática e/ou de fatores ambientais presentes na área de estudo que podem influenciar sua biodisponibilidade (CCME, 1999a). Preocupação semelhante com o $\mathrm{Zn}$ foi verificada na bacia do rio Tietê, onde a concentração deste metal mostrou-se moderada/forte em referência a poluição (MORTATTI; HISSLER; PROBST, 2010).

O uso dos VGQS sem levar em conta os valores referências das substâncias ou testes de toxicidade pode levar a conclusões não verdadeiras sobre a qualidade dos sedimentos. Sendo assim, apesar de relevante esses valores devem ser utilizados com cuidado na tomada de decisões para a proteção ou melhoria da qualidade dos sedimentos. Segundo Fagnani et al. (2011), os parâmetros físicos, químicos e toxicológicos da água e do sedimento permitem apenas mimetizar os ecossistemas aquáticos naturais, uma vez que a interação entre os compartimentos é complexa e dinâmica pois, além de processos naturais ou artificiais de ressuspensão também os organismos bentônicos que vivem na porção superior das camadas de sedimentos de fundo interferem nos fluxos na interface sedimento/coluna d'água.

Ao se comparar as concentrações de metais nos sedimentos fluviais amostrados com outros estudos pode-se observar que no geral os teores obtidos estão abaixo da maioria encontrada mundialmente em rios (Tabela 2). Mohiuddin et al. (2011) em estudos de metais em sedimentos do rio Buriganga em Bangladesh sujeito principalmente a poluição industrial e resíduos domésticos, Woitke et al. (2003) em análises dos sedimentos no rio Danúbio na Hungria que sofrem influência de poluentes industriais, agrícolas e domésticos, e Massolo et al. (2012) em sedimentos do rio Hugli na Índia que sofrem ações de indústrias, encontraram valores mais elevados para o $\mathrm{Cd}, \mathrm{Cu}$ e $\mathrm{Cr}$ do que aqueles encontrados em nosso estudo. Concentrações de $\mathrm{Pb}$ inferiores às obtidas neste estudo foram encontradas apenas no rio Buriganga em Bangladesh (MOHIUDDIN et al., 2011) e concentrações muito superiores foram encontradas no rio Delta na China que sofre influência de efluentes industriais (CHEUNG et al., 2003). Para o Fe, nosso estudo mostra concentrações abaixo daquelas obtidas em sedimentos dos rios Danúbio na Hungria (WOITKE et al., 2003), rio Hugli na Índia (MASSOLO et al., 2012) e Saricary na Turquia que sofre influência de resíduos de pequenas indústrias, atividades agrícolas e efluentes domésticos (LEVENT TUNA et al., 2007). Para o Zn concentrações mais elevadas do que aquelas reportadas em nosso estudo foram encontradas apenas no rio Saricary na Turquia (LEVENT TUNA et al., 2007).

Quando se compara com rios brasileiros (Tabela 2), Depiné et al. (2012) ao estudarem os sedimentos fluviais de uma bacia agrícola em Lontras (SC) obteve teores de Cd inferiores, $\mathrm{Zn}$ cerca da metade da concentração e $\mathrm{Cu}, \mathrm{Pb}$ e Fe muito acima dos valores observados em nosso estudo. Ao analisar sedimentos do rio Pitimbu no Rio Grande do Norte, também sujeito a fontes de contaminação antrópicas vindas da agricultura, Santos, Melo Júnior e Segundo (2002) obtiveram teores de $\mathrm{Zn}$ inferiores, de Fe semelhantes e de $\mathrm{Cr}, \mathrm{Cu}, \mathrm{Pb}$ e $\mathrm{Mn}$ mais altos que em nosso estudo. Mortatti, Hissler e Probst (2010) analisaram metais em sedimentos fluviais em sete pontos do curso principal do rio Tietê localizados de sua nascente até a barragem de Barra Bonita. Os autores verificaram concentrações superiores de $\mathrm{Cr}, \mathrm{Cu}, \mathrm{Pb}$; também para o $\mathrm{Zn}$, exceto no ponto das nascentes, as concentrações foram superiores as desse estudo, no entanto, deve-se considerar que o rio Tietê está sujeito à contaminação por efluentes domésticos, industriais e agrícolas. Os valores encontrados em nosso estudo para $\mathrm{Cr}$, $\mathrm{Cu}$ e Mn são inferiores e de $\mathrm{Zn}$ mais elevados do que os obtidos em sedimentos dos Rios Feitoria e Cadeia no Rio Grande do Sul, (RODRIGUES; FORMOSO, 2006). Saraiva et al. (2009) ao analisar sedimentos do ribeirão Espírito Santo, afluente direto do rio São Francisco na região de Três Marias em Minas Gerais, também encontraram valores inferiores para $\mathrm{Zn}$ e semelhantes para $\mathrm{Cu}$ em relação ao nosso estudo.

Outro resultado interessante é as concentrações de Mn e Fe estarem abaixo do esperado, uma vez que os Latossolos que fornecem sedimentos aos cursos d'água estudados apresentam usualmente teores elevados nesses metais (MARQUES et al., 2004). Todavia, isso pode ser explicado quando se leva em consideração que os óxidos de ferro e manganês em ambientes anóxicos, como aqueles reinantes no fundo de cursos fluviais, podem ser reduzidos a formas mais solúveis e facilitar sua remoção dos sedimentos (KRUPADAM; SMITA; WATE., 2006). 
Tabela 2 - Média ou faixa de concentrações mundiais e nacionais de metais poluentes nos sedimentos fluviais $\left(\mu \mathrm{g} . \mathrm{g}^{-1}\right)$.

\begin{tabular}{|c|c|c|c|c|c|c|c|c|}
\hline $\begin{array}{c}\text { Área } \\
\text { Geográfica }\end{array}$ & Cd & Mn & $\mathbf{P b}$ & $\mathrm{Cu}$ & $\mathrm{Zn}$ & $\mathbf{C r}$ & $\mathrm{Fe}$ & Referência \\
\hline $\begin{array}{c}\text { Rio Buriganga, } \\
\text { Bangladesh }\end{array}$ & 0,22 & & 0,23 & 2,74 & 0,22 & 1,96 & & $\begin{array}{c}\text { MOHIUDDIN } \\
\text { et al., } 2011\end{array}$ \\
\hline $\begin{array}{c}\text { Rio Delta, } \\
\text { China }\end{array}$ & $7,2-7,8$ & & $166,7-264,2$ & $49,3-69,0$ & - & - & - & CHEUNG et al., 2003 \\
\hline $\begin{array}{c}\text { Rio Danúbio, } \\
\text { Hungria }\end{array}$ & 1,2 & & 46,3 & 65,7 & - & $26,5-556,5$ & $17,600-64,600$ & WOITKE et al., 2003 \\
\hline $\begin{array}{l}\text { Rio Hugli, } \\
\text { Índia }\end{array}$ & $0,03-1,79$ & & $17,0-33,2$ & $22,1-36,8$ & $64,1-90,7$ & $58,2-76,8$ & $33,43-40,83$ & MASSOLO et al., 2012 \\
\hline $\begin{array}{c}\text { Rio Saricay, } \\
\text { Turquia } \\
\end{array}$ & - & & $0,7-31,3$ & $7-128$ & $18-304$ & $15-1308$ & $1120-13210$ & $\begin{array}{c}\text { LEVENT TUNA } \\
\text { et al., } 2007\end{array}$ \\
\hline $\begin{array}{c}\text { Ribeirão } \\
\text { Concórdia, } \\
\text { Brasil, SC } \\
\end{array}$ & - & - & 37,0 & 48,33 & 110,00 & 16,67 & 2742,19 & DEPINÉ et al., 2012 \\
\hline $\begin{array}{c}\text { Rio Pitimbu, } \\
\text { Brasil, RN }\end{array}$ & - & 29,33 & 21,00 & 18,67 & 28,44 & 54,56 & - & SANTOS et al., 2002 \\
\hline $\begin{array}{l}\text { Rio Tietê, } \\
\text { Brasil, SP }\end{array}$ & - & - & $18-77$ & $39-200$ & $88-648$ & $60-262$ & - & MORTATTI et al., 2010 \\
\hline $\begin{array}{c}\text { Rio Feitoria e } \\
\text { Cadeia, } \\
\text { Brasil, RS } \\
\end{array}$ & - & $1080-2230$ & - & $57,8-102$ & $126-255$ & $34,7-1031$ & $8680-10300$ & $\begin{array}{c}\text { RODRIGUES e } \\
\text { FORMOSO, } 2006\end{array}$ \\
\hline $\begin{array}{c}\text { Ribeirão } \\
\text { Espírito Santo, } \\
\text { Brasil, MG } \\
\end{array}$ & 0,001 & - & $0,0016-0,01$ & $0,004-0,009$ & $0,02-0,18$ & - & - & SARAIVA et al., 2009 \\
\hline $\begin{array}{c}\text { Bacia do rio } \\
\text { Meia Ponte, } \\
\text { Brasil, GO } \\
\end{array}$ & $\begin{array}{c}0,0374(\mathrm{M}) \\
0,0394(\mathrm{~J})\end{array}$ & $\begin{array}{c}3,5600(\mathrm{M}) \\
3,1350(\mathrm{~J})\end{array}$ & $\begin{array}{c}0,5530(\mathrm{M}) \\
0,5530(\mathrm{~J})\end{array}$ & $\begin{array}{c}3,2800(\mathrm{M}) \\
2,8430(\mathrm{~J})\end{array}$ & $\begin{array}{c}181,27(\mathrm{M}) \\
179,93(\mathrm{~J})\end{array}$ & $\begin{array}{c}0,8930(\mathrm{M}) \\
0,8860(\mathrm{~J})\end{array}$ & $\begin{array}{c}2,0440(\mathrm{M}) \\
1,7900(\mathrm{~J})\end{array}$ & Este estudo \\
\hline
\end{tabular}

Fonte: adaptado de Mohiuddin et al. (2011)

(M) - montante; (J) jusante

As diferenças entre as concentrações dos metais nos sedimentos amostrados são influenciadas pelo fator bacia hidrográfica $(\mathrm{F}(16,104)=6,7957$, $\mathrm{p}=0,00001$ ) (Gráfico 1). Isto é devido principalmente às maiores concentrações dos metais na bacia do ribeirão Santa Maria em relação a bacia do rio Meia Ponte para o $\mathrm{Al}(\mathrm{p}=0,00650)$; dos rios Meia Ponte e Piracanjuba para o Cd $(p=0,00014$ e $p=0,00039$, respectivamente $), \mathrm{Cr}(\mathrm{p}=0,00012$ e $\mathrm{p}=0,00024$, respectivamente $), M n(p=0,00013$ e $p=0,00012$, respectivamente) e $\mathrm{Zn}(\mathrm{p}=0,00023$ e $\mathrm{p}=0,00012$, respectivamente); e da bacia do rio Piracanjuba em relação a do rio Meia Ponte para o Mn ( $\mathrm{p}=0,00092)$ (Tabela 3).
Fatores geológicos podem explicar as diferenças observadas no comportamento dos metais em cada bacia hidrográfica. Os cursos fluviais amostrados na bacia do ribeirão Santa Maria drenam rochas basálticas pertencentes ao Grupo São Bento enquanto os cursos fluviais amostrados na bacia do rio Meia Ponte drenam ortognaisses de composição granítica pertencentes ao Complexo Plutônico do Arco Magmático de Goiás e aqueles amostrados na bacia do rio Piracanjuba drenam xistos e paragnaisses (SGM, 2004). Trabalhos anteriores mostram que os basaltos que ocorrem na área de estudo são enriquecidos em $\mathrm{Cr}, \mathrm{Cu}$, Mn e $\mathrm{Zn}$ e possuem menor teor de $\mathrm{Al}$ em relação aos ortognaisses graníticos, paragnaisses e xistos (NAVARRO; ZANARDO, 2007; NA- 


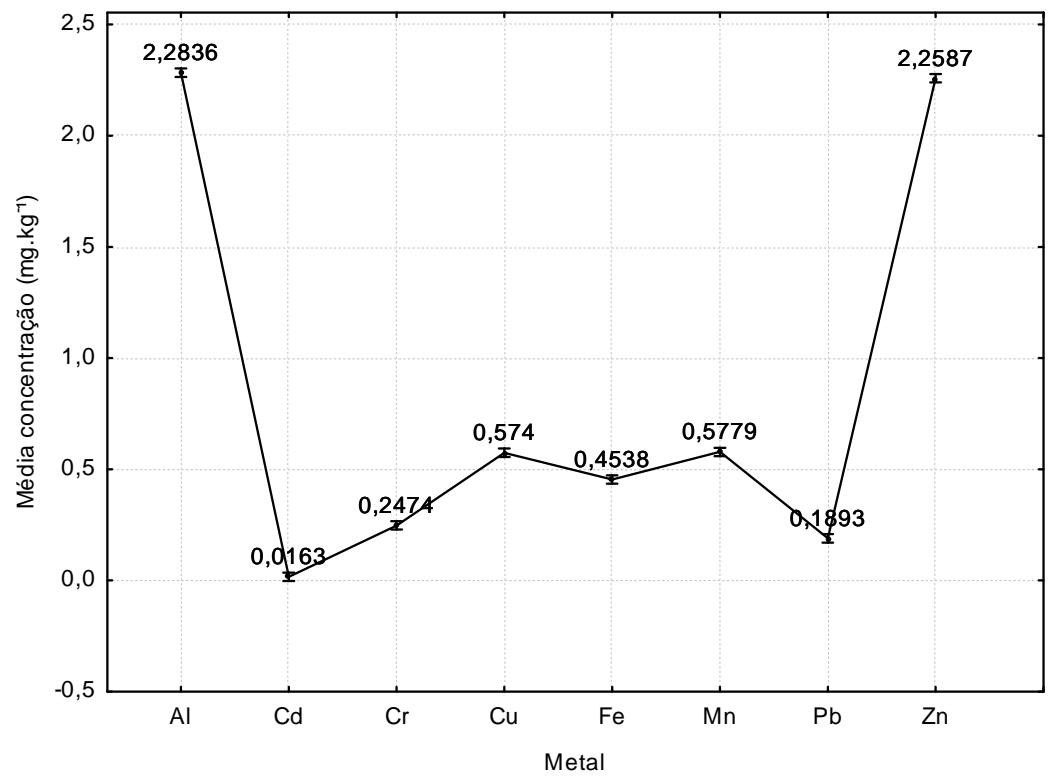

Gráfico 1 - Resultados da ANOVA one-way relativos à comparação entre as médias $(\log \mathbf{x}+1)$ das concentrações dos metais nos sedimentos fluviais amostrados e agrupados por bacia hidrográfica. As barras verticais indicam o desvio padrão

Tabela 3 - Resultados do teste de Tukey da comparação das concentrações dos metais nos sedimentos fluviais amostrados agrupados por bacia hidrográfica. Os valores em negrito indicam diferenças significativas $(p<0,05)$

\begin{tabular}{|c|c|c|c|c|}
\hline Metal & Bacia & Meia Ponte & Piracanjuba & Santa Maria \\
\hline \multirow[t]{3}{*}{$\mathrm{Al}$} & Meia Ponte & 1,00000 & & \\
\hline & Piracanjuba & 0,36497 & 1,00000 & \\
\hline & Santa Maria & 0,00650 & 0,18305 & 1,00000 \\
\hline \multirow[t]{3}{*}{$\mathrm{Cd}$} & Meia Ponte & 1,00000 & & \\
\hline & Piracanjuba & 0,88571 & 1,00000 & \\
\hline & Santa Maria & 0,00014 & 0,00039 & 1,00000 \\
\hline \multirow[t]{3}{*}{$\mathrm{Cr}$} & Meia Ponte & 1,00000 & & \\
\hline & Piracanjuba & 0,60214 & 1,00000 & \\
\hline & Santa Maria & 0,00012 & 0,00024 & 1,00000 \\
\hline \multirow[t]{3}{*}{$\mathrm{Cu}$} & Meia Ponte & 1,00000 & & \\
\hline & Piracanjuba & 0,59518 & 1,00000 & \\
\hline & Santa Maria & 0,99974 & 0,65103 & 1,00000 \\
\hline \multirow[t]{3}{*}{$\mathrm{Fe}$} & Meia Ponte & 1,00000 & & \\
\hline & Piracanjuba & 0,65905 & 1,00000 & \\
\hline & Santa Maria & 0,65788 & 0,99968 & 1,00000 \\
\hline \multirow[t]{3}{*}{$\mathrm{Mn}$} & Meia Ponte & 1,00000 & & \\
\hline & Piracanjuba & 0,00092 & 1,00000 & \\
\hline & Santa Maria & 0,00013 & 0,00012 & 1,00000 \\
\hline \multirow[t]{3}{*}{$\mathrm{Pb}$} & Meia Ponte & 1,00000 & & \\
\hline & Piracanjuba & 0,91832 & 1,00000 & \\
\hline & Santa Maria & 0,27985 & 0,51102 & 1,00000 \\
\hline \multirow[t]{3}{*}{$\mathrm{Zn}$} & Meia Ponte & 1,00000 & & \\
\hline & Piracanjuba & 0,46943 & 1,00000 & \\
\hline & Santa Maria & 0,00023 & 0,00012 & 1,00000 \\
\hline
\end{tabular}




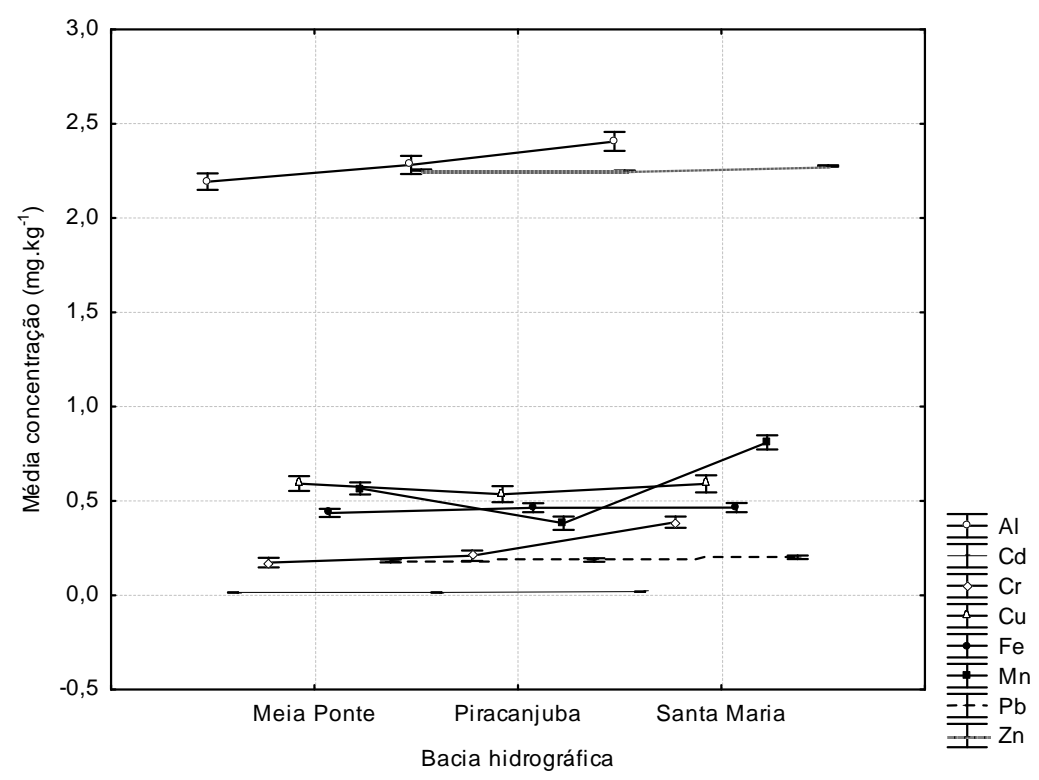

Gráfico 2 - Comparação entre as médias $(\log x+1)$ das concentrações dos metais nos sedimentos fluviais amostrados resultantes da ANOVA one-way. As barras verticais indicam o desvio padrão

Tabela 4 - Matriz resultante do teste de Tukey referente à comparação entre as concentrações de metais nos sedimentos fluviais amostrados. Os valores em negrito indicam diferenças significativas $(\mathbf{p}<0,05)$

\begin{tabular}{lllllllll}
\hline & $\mathrm{Al}$ & $\mathrm{Cd}$ & $\mathrm{Cr}$ & $\mathrm{Cu}$ & $\mathrm{Fe}$ & $\mathrm{Mn}$ & $\mathrm{Pb}$ & $\mathrm{Zn}$ \\
\hline $\mathrm{Al}$ & 1,00000 & & & & & & & \\
$\mathrm{Cd}$ & $\mathbf{0 , 0 0 0 0 3}$ & 1,00000 & & & & & & \\
$\mathrm{Cr}$ & $\mathbf{0 , 0 0 0 0 3}$ & $\mathbf{0 , 0 0 0 0 3}$ & 1,00000 & & & & & \\
$\mathrm{Cu}$ & $\mathbf{0 , 0 0 0 0 3}$ & $\mathbf{0 , 0 0 0 0 3}$ & $\mathbf{0 , 0 0 0 0 3}$ & 1,00000 & & & & \\
$\mathrm{Fe}$ & $\mathbf{0 , 0 0 0 0 3}$ & $\mathbf{0 , 0 0 0 0 3}$ & $\mathbf{0 , 0 0 0 0 3}$ & $\mathbf{0 , 0 0 0 2 1}$ & 1,00000 & & & \\
$\mathrm{Mn}$ & $\mathbf{0 , 0 0 0 0 3}$ & $\mathbf{0 , 0 0 0 0 3}$ & $\mathbf{0 , 0 0 0 0 3}$ & 1,00000 & $\mathbf{0 , 0 0 0 1 2}$ & 1,00000 & & \\
$\mathrm{~Pb}$ & $\mathbf{0 , 0 0 0 0 3}$ & $\mathbf{0 , 0 0 0 0 3}$ & 0,36909 & $\mathbf{0 , 0 0 0 0 3}$ & $\mathbf{0 , 0 0 0 0 3}$ & $\mathbf{0 , 0 0 0 0 3}$ & 1,00000 & \\
$\mathrm{Zn}$ & 0,98324 & $\mathbf{0 , 0 0 0 0 3}$ & $\mathbf{0 , 0 0 0 0 3}$ & $\mathbf{0 , 0 0 0 0 3}$ & $\mathbf{0 , 0 0 0 0 3}$ & $\mathbf{0 , 0 0 0 0 3}$ & $\mathbf{0 , 0 0 0 0 3}$ & 1,00000 \\
\hline
\end{tabular}

VARRO et al., 2011; RENNER, 2010). Por exemplo, a concentração média de $\mathrm{Cr}$ nos basaltos é de 50 mg.kg-1 , Cu 190 mg.kg-1 ${ }^{-1}$ Mn 0,20 wt.\% e Al 12 wt.\% enquanto nos ortognaisses graníticos é de $0 \mathrm{mg} \cdot \mathrm{kg}^{-1}$ para $\mathrm{Cr}, 15 \mathrm{mg} . \mathrm{kg}^{-1}$ para $\mathrm{Cu}, 0,05$ wt. $\%$ para $\mathrm{Mn}$ e 15 wt.\% para Al (NAVARRO; ZANARDO, 2007; RENNER, 2010). Assim, concentrações mais elevadas de Cr, Mn e Zn encontradas para os pontos amostrados na bacia do ribeirão Santa Maria podem ser explicadas pela composição geoquímica dos basaltos e solos associados presentes na área, os quais por serem enriquecidos nesses metais constituem áreas fontes de sedimentos para as águas dos cursos fluviais. Ainda não é claro porque em nosso estudo $\mathrm{Cu}$ não sofre interferência direta dos fatores geológicos, tal como ocorre com $\mathrm{Cr}$, Mn e Zn. Outras pesquisas devem ser desenvolvidas para elucidar essa questão.

Ao se comparar as concentrações dos metais entre si, a análise ANOVA one-way indica que existem diferenças significativas entre as concentrações dos metais amostrados $(\mathrm{F}(7, \quad 488)=2334,6$, $\mathrm{p}=0,00001)$, com destaque para as elevadas concentrações médias de $\mathrm{Al}$ e $\mathrm{Zn}$, e para a menor concentração média de Cd (Gráfico 2). Estas diferenças ocorrem entre os metais considerados, exceto entre $\mathrm{Cu}$ e $\mathrm{Mn}(\mathrm{p}=1,00000)$, Al e $\mathrm{Zn}(\mathrm{p}=0,98324)$ e $\mathrm{Cr}$ e $\mathrm{Pb}$ $(p=0,36909) \quad$ (Tabela 4). A diferença significativa entre as concentrações dos metais indica que os sedimentos estudados estão expostos a diferentes fontes de poluição que podem ser de origem natural 
Tabela 5 - Estatísticas do teste de " $t$ " comparando as concentrações de metais nos sedimentos fluviais amostrados por posição (montante, jusante) em relação às aguadas. Os valores em negrito indicam diferenças significativas $(p<0,05)$.

$\mathrm{DP}=$ desvio padrão; $\mathrm{n}=$ número de amostras; gl=graus de liberdade

\begin{tabular}{llllllll}
\hline Metal & Posição & Média $\log (\overline{\mathbf{x}}+\mathbf{1})$ & DP & n & t & gl & p \\
\hline $\mathrm{Al}$ & Montante & 2,28855 & 0,24208 & & & & \\
& Jusante & 2,27233 & 0,23526 & 28 & 0,73126 & 27 & 0,47092 \\
\hline $\mathrm{Cd}$ & Montante & 0,01598 & 0,00334 & & & & \\
& Jusante & 0,01672 & 0,00374 & 28 & $-1,30813$ & 27 & 0,20186 \\
\hline $\mathrm{Cu}$ & Montante & 0,59894 & 0,16560 & & & & \\
& Jusante & 0,52901 & 0,22032 & 28 & 1,60763 & 27 & 0,11955 \\
\hline $\mathrm{Cr}$ & Montante & 0,24647 & 0,16137 & & & & \\
& Jusante & 0,24447 & 0,14520 & 28 & 0,10100 & 27 & 0,92029 \\
\hline $\mathrm{Fe}$ & Montante & 0,47335 & 0,08627 & & & & \\
& Jusante & 0,44079 & 0,11632 & 28 & 1,38620 & 27 & 0,17703 \\
\hline $\mathrm{Mn}$ & Montante & 0,18741 & 0,04034 & & & & \\
& Jusante & 0,18786 & 0,03738 & 28 & $-0,94845$ & 27 & 0,94845 \\
\hline $\mathrm{Pb}$ & Montante & 0,60190 & 0,23929 & & & & \\
& Jusante & 0,53980 & 0,23462 & 28 & 1,96336 & 27 & 0,05998 \\
\hline $\mathrm{Zn}$ & Montante & 2,25918 & 0,01965 & & & & \\
& Jusante & 2,25563 & 0,02005 & 28 & 2,176626 & 27 & $\mathbf{0 , 0 3 8 4 3}$ \\
\hline
\end{tabular}

ou antrópica. No presente caso, acredita-se que a fonte destes metais seja natural e associada a fatores geológicos e pedológicos tais como tipos de rochas e solos.

Quando comparadas as concentrações das amostras de sedimentos coletadas a montante e a jusante das aguadas, o teste de " $\mathrm{t}$ " indica que há diferenças significativas para o $\mathrm{Zn}(\mathrm{p}=0,03843)$, com os valores da concentração mais elevados a montante que a jusante (Tabela 5). Era de se esperar que as concentrações de todos os metais analisados fossem mais elevadas a jusante caso houvesse contribuições antrópicas provenientes da atividade pecuária leiteira exercida na região que utiliza as aguadas para dessedentação do gado. No entanto, o resultado obtido para o $\mathrm{Zn}$ foi inverso ao esperado e não está claro porque isso acontece. Estudos complementares deverão ser realizados para elucidar essa questão.

\section{CONCLUSÕES}

Pode-se concluir que com exceção do Zn e $\mathrm{Al}$ todos os metais analisados encontram-se em concentrações baixas quando comparadas com outras regiões semelhantes e não apresentam risco a biota. O Zn apresenta concentração possível de causar danos ao ambiente aquático, entretanto a determi- nação da concentração natural e de sua toxicidade são recomendados antes de se fazer qualquer afirmação quanto ao efeito poluidor desse metal.

As concentrações dos metais, com exceção do $\mathrm{Zn}$, não foram significativamente diferentes a montante e jusante o que demonstra não haver efeito antrópico, ou seja, na região estudada a criação de gado não contribui para a poluição dos recursos hídricos.

Finalmente, pode-se afirmar que as concentrações dos metais são influenciadas pela bacia hidrográfica, especificamente por sua geologia.

\section{AGRADECIMENTOS}

Os autores agradecem ao CNPq e FAPEG pelo auxílio financeiro

\section{REFERÊNCIAS}

ABDEL-GHANI, N. T.; ELCHAGHABY, G. A. Influence of operating conditions on the removal of $\mathrm{Cu}$, $\mathrm{Zn}$, Cd and $\mathrm{Pb}$ ions from wastewater by adsorption. International Journal Environmental Science Technology, v. c4, n. 4, p. 451-456, 2007. 
ADAMS, W. J.; KIMERLE, R. A.; BARNETT, J. W. Sediment quality and aquatic life assessment. Environmental Science Technology, v. 26, n. 10, p. 1865$1875,1992$.

ADEYEMO, O. K.; ADEDOKUN, O. A.; YUSUF, R. K.; ADELEYE, E. A. Seasonal changes in physicaochemical parameters and nutrient load of river sediment in Ibadan city, Nigeria. Global NEST Journal, v. 10, n. 3, p. 326-336, 2008.

AKCAY, H.; OGUZ, A.; KARAPIRE, C. Study of heavy metal pollution and speciation in Buyak Menderes and Gediz river sediments. Water Research, v. 37, n. 4, p. 813-822, 2003.

ALLOWAY, B. J. Heavy metals in soils. London: Blackie Academic \& Professional, 1995.

BRASIL. Conselho Nacional do Meio Ambiente. Resolução CONAMA $n^{o}$ 454, de 01/11/2012. Disponível em: <http://www.mma.gov.br/port/conama/ legiabre.cfm?codlegi=693>. Acesso em: 08 jul. 2013.

CAMPBELL, P. G. C.; TESSIER, A. Ecotoxicology of metals in aquatic environments: geochemical aspects. In: NEWMAN, M. C.; JAGOE, C. H. (Ed.). Ecotoxicology: a hierarchical treatment. Boca Raton, FL: Lewis Publishers, 1996.

CCME. Environmental CANADA Canadian Council of Ministers of the Environment. Protocol for the derivation of Canadian sediment quality guidelines for the protection of aquatic life. CCME EPC-98E. 1995. Prepared by Environment Canada, Guidelines Division, Technical Secretariat of the CCME Task Group on Water Quality Guidelines, Ottawa. (Reprinted in Canadian environmental quality guidelines, Chapter 6, Canadian Council of Ministers of the Environment, Winnipeg, 1999a).

CCME. Canadian Council of Ministers of the Environment. Canadian sediment quality guidelines for the protection of aquatic life: Zinc. In: CANADIAN COUNCIL OF MINISTERS OF THE ENVIRONMENT. Canadian environmental quality guidelines. Winnipeg: Canadian Council of Ministers of the Environment, 1999b.

CEREJEIRA, M .J.; VIANA, P.; BATISTA, S. Pesticides in portuguese surface and ground waters. $W a$ ter Research, v. 37, n. 5, p. 1055-1063, 2003.
CHEUNG, K. C.; POON, B. H. T.; LAN, C. Y.; WONG, M. H. Assessment of metal and nutrient concentrations in river water and sediment collected from the cities in the Pearl River Delta, South China. Chemosphere, v. 52, n. 9, p. 1431-1440, 2003.

DAVIES, O. A.; ABOWEI, J. F. N. Sediment quality of lower reaches of Okpoka Creek, Niger Delta, Nigeria. European Journal of Scientific Research, v. 26, n. 3, p. 437-442, 2009.

DEPINÉ, H.; KAUFMANN, V.; PINHEIRO, A.; SILVA, M. R.; GONÇALVES Jr. A. C.; GOMÊS, G. D. Nutrientes e metais em sedimentos depositados no rio de uma bacia agrícola no estado de Santa Catarina. Revista Brasileira de Recursos Hídricos, v. 17, n. 4, p. 229-237, 2012.

DOWNEY, N.E.; MOORE, J. F. Trichostrongylid contamination of pasture fertilized with cattle slurry. Veterinary Record, v. 101, n. 24, p. 487-488, 1977.

EBRAHIMI, M.; TAHERIANFARD, M. Pathological and Hormonal Changes in Freshwater Fishes Duo to Exposure to Heavy Metals Pollutants. Water, Air, and Soil Pollution, v. 217, n. 1-4, p. 47-55, 2011.

FAGNANI, E.; GUIMARÃES, J. R.; MOZETO, A. P.; FADINI, P. S. Sulfetos volatilizáveis por acidificação e metais extraídos simultaneamente na avaliação de sedimentos de água doce. Química Nova, v. 34, n. 9, p. 1618-1628, 2011.

FAO. FAOSTAT. Produção mundial de leite - 20002010. [S.l.]: FAO; 2012.

FITCH, L.; ADAMS, B. W. Can cows and fish coexist? Canadian Journal of Plant Science, v. 78, p. 191198, 1998.

IBGE. Diretoria de Pesquisas, Coordenação de Agropecuária. Pesquisa da Pecuária Municipal. [S.l.]: IBGE, 2011.

IMB. Instituto Mauro Borges. Disponível em: $<$ http://www.seplan.go.gov.br/sepin/ $\geq$. Acesso em: 05 out. 2012.

JESUS, H. C.; COSTA, E. A.; MENDONCA, A. S. F.; ZANDONADE, E. Distribuição de metais pesados em sedimentos do sistema estuarino da Ilha de Vitória-ES. Química Nova, v. 27, n. 3, p. 378-386, 2004. 
KRUPADAM, R. J; SMITA, P.; WATE, S. R. Geochemical fractionation of heavy metals in sediments of the Tapi estuary. Geochemical Journal, v. 40, n. 5, p. 513-522, 2006.

LEVENT TUNA, A.; YILMAZ, F.; DEMIRAK, A.; OZDEMIR, N. Sources and distribution of trace metals in the Saricay stream basin of southwestern Turkey. Environmental Monitoring and Assessment, v. 125, n. 1-3, p. 47-57, 2007.

LIM, T. T.; EDWARDS, D. R.; WORKMAN, S. R.; LARSON, B. T.; DUNN, L. Vegetated filter strip removal of cattle manure constituents in runoff. Transactions of the American society of Agricultural and Biological Engineers, v. 41, n. 5, p. 1375-1381, 1998.

MARÇAL, W. S.; GASTE, L.; NASCIMENTO, M. R. L.; LIBONI, M.; GOMES, G. P.; HISASI, C. S. Cadmium concentration in mineral salt mixtures used as supplementation in beef cattle food. Veterinarski Arhiv, Zagreb, v. 73, n. 1, p. 47-53, 2003.

MARQUES, J. J; SCHULZE, D. G; CURI, N; MERTZMAN, S. A. Trace element geochemistry in Brasilian Cerrado soils. Geoderma, v. 121, n. 1-2, p. 3143, 2004.

MASSOLO, S.; BIGNASCA, A.; SARKAR, S. K.; CHATTERJEE, M.; ATTACHARYA, B. D; ALAM, A. Geochemical fractionation of trace elements in sediments of Hugli River (Ganges) and Sundarban wetland (West Bengal, India). Environmental Monitoring and Assessment, v. 184, n. 12, p. 7561-7577, 2012.

MOHIUDDIN, K. M.; OGAWA, Y.; ZAKIR, H. M.; OTOMO, K.; SHIKAZONO, N. Heavy metals contamination in the water and sediments of an urban river in a developing country. International Journal Environmental Science Technology, v. 8, n. 4, p. 723-736, 2011.

MORGANO, M. A.; GOMES, P. C.; MANTOVANI, D. M. B.; PERRONE, A. A. M.; SANTOS, T. F. Níveis de mercúrio total em peixes de água doce de pisciculturas paulistas. Ciência e Tecnologia de Alimentos, v. 25, n. 2, p. 250-253, 2005.

MORILLO, J.; USERO, J.; GRACIA, I. Heavy metal distribution in marine sediments from the southwest coast of Spain. Chemosphere, v. 55, n. 3, p. 431-442, 2004.
MORTATTI, J.; HISSLER, C.; PROBST, J-L. Distribuição de metais pesados nos sedimentos de fundo ao longo da Bacia do rio Tietê. Geologia USP. Série Científica, v. 10, n. 2, p. 3-11, 2010.

MUNIZ, D. H. F.; OLIVEIRA-FILHO, E. C. Metais pesados provenientes de rejeitos de mineração e seus efeitos sobre a saúde e o meio ambiente. Universitas: Ciências da Saúde, v. 4, n. 1-2, p. 83-100, 2006.

NAVARRO, G. R. B.; ZANARDO, A. Geoquímica de Gnaisses do arco magmático de Goiás na Região Sul do Estado de Goiás. Geologia USP. Série Científica, v. 7, n. 1, p. 19-28, 2007.

NAVARRO, G. R. B.; ZANARDO, A.; CONCEIÇÃO, F. T.; MORAES, R.; SIMÕES, L. S. A. Química mineral e estimativas de pressão e temperatura em rochas metassedimentares do Grupo Araxá na região de Morrinhos, Sul do estado de Goiás. Geologia USP. Série Científica, v. 11, n. 2, p. 3-20, 2011

NOURI, J.; LORESTANI, B.; YOUSEFI, N.; KHORASANI, N.; HASANI, A. H.; SEIF, S.; CHERAGHI, M. Phytoremediation potential of native plants grown in the vicinity of Ahangaran leadzinc mine (Hamedan, Iran). Environmental Earth Science, v. 62, n. 3, p. 639-644, 2011.

OLADE, M. A. Heavy metal pollution and the need for monitoring: illustrated for developing countries in West Africa. In: HUTCHINSON, T.; MEEMA, K. (Ed.). Lead, Mercury, Cadmium and Arsenic in the environment. Chichester: John Wiley, 1987. p. 335-341.

OLUBUNMI, F, E.; OLORUNSOLA, O. E. Evaluation of the Status of Heavy Metal Pollution of Sediment of Agbabu Bitumen Deposit Area. European Journal of Scientific Research, v. 41, n. 3, p. 373-382, 2010.

ORRICO JÚNIOR, M. A. P.; ORRICO, A. C. M.; LUCAS JUNIOR, J.; SAMPAIO, A. A. M.; FERNANDES, A. R. M.; OLIVEIRA, E. A. Compostagem dos dejetos da bovinocultura de corte: influência do período, do genótipo e da dieta. Revista Brasileira de Zootecnia, v. 41, n. 5, p. 1301-1307, 2012.

PRAVEENA, S. M.; RADOJEVIC, M.; ABDULLAH, M. H.; AVIS, A. Z. Factor-cluster analysis and enrichment study of mangrove sediments - An example from Mengkabong Sabah. Malysian Journal of Analytical Sciences, v. 2, n. 6, p. 421-430, 2007. 
RENNER, L. C. Geoquímica de sills basálticos da Formação Serra Geral, sul do Brasil, com base em rocha total e micro-análise de minerais. 2010. $226 \mathrm{f}$. Tese (Doutorado) - Instituto de Geociências, Universidade Federal do Rio Grande do Sul, Santa Catarina, 2010.

RODRIGUES, M. L. K.; FORMOSO, M. L. L. Geochemical distribution of selected heavy metals in stream sediments affected by tannery activities. Water, Air, and Soil Pollution, v. 169, n. 1-4, 167-184, 2006.

SANTOS, A. D. R. L; MELO JÚNIOR, G.; SEGUNDO, J. E. A. G. Concentração de metais pesados em frações granulométricas de sedimentos de fundo do rio Pitimbu, região sul da grande $\operatorname{Natal}(\mathrm{RN})$ : Implicações para levantamentos ambientais. Revista de Geologia, v. 15, p. 1-8, 2002.

SARAIVA, V. K.; NASCIMENTO, M. R. L; PALMIERI, H. E. L; JACOMINI, V. M. F. Avaliação da qualidade de sedimentos - estudo de caso: sub-bacia do ribeirão Espírito Santo, afluente do rio São Francisco. Química Nova, v. 32, n. 8, p. 1995-2002, 2009.

SHIGAKI, F.; SHAPPLEY, A.; PROCHONOW, L .I. Animal-based agriculture phosphorus management and water quality in Brazil: options for the future. Scientia Agricola, v. 63, n.2, p. 194-209, 2006.

SGM. Superintendência de Geologia e Minerais de Goiás. Mapa geológico do Estado de Goiás e Distrito Federal associado a modelo digital de terreno. Goiânia: Secretaria de Indústria e Comércio de Goiás, Brasil, 2004.

SUCIU, I.; COSMA, C.; TODICA, M.; BOLBOACA, S. D.; JANTSCHI, L. Analysis of soil heavy metal pollution and pattern in Central Transylvania. Internationa Journal of Molecular Sciences, v. 9, p. 434453, 2008.

TESSIER, A.; CAMPBELL, P. G. C. Partitioning of trace metals in sediments: relationship with bioavailability. Hydrobiologia, v. 149, n. 1, p. 43-52, 1987.

TIMOFIECSYK, A.; FAVARETTO, N.; PAULETTI, V.; DIRCKOW, J. Perdas de carbono e nitrogênio com aplicação de dejeto líquido bovino em latossolo muito Argiloso sob plantio direto e chuva natural. Revista Brasileira de Ciência do Solo, v. 36, n. 6, p. 19241930, 2012.
USEPA. UNITED STATES EPA. Method 3050B, SW846. Test methods for evaluating solid waste: physical/chemical methods. 3rd ed. USA: USEPA, 1995.

VRIES, W.; ROMKENS, P. F. A. M.; SCHUTZE, G. Critical soil concentrations of cadmium, lead and mercury in view of health effect on humans and animals. Reviews of Environmental Contamination and Toxicology, v. 191, p. 91-30, 2007.

WOITKE, P.; WELLMITZ, J.; HELM, D.; KUBE, P.; LEPOM, P.; LITHERATY, P. Analysis and assessment of heavy metal pollution in suspended solids and sediments of the river Danube. Chemosphere, v. 51, n. 8, p. 633-642, 2003.

\section{Pollutant Metals In Fluvial Sediments From Creeks Under The Influence Of Dairy Activities In The State of Goiás, Brazil}

\section{ABSTRACT}

Level and source of contamination of aquatic ecosystems are usually determined by the concentration of pollutant metals in sediments. This study aimed to determine the effects of dairy activities on the contamination of sediments by $\mathrm{Cu}, \mathrm{Fe}, \mathrm{Mn}, \mathrm{Zn}, \mathrm{Cd}, \mathrm{Pb}, \mathrm{Cr}$ and Al. Samples were collected upstream and downstream from cattle watering points in 31 creeks located in the Meia Ponte river region, which is the main dairy area in the State of Goiás, Brazil. The metals were analyzed by atomic absorption at sediment fractions $<0.063 \mathrm{~mm}$ after acid digestion. Except for $\mathrm{Al}$ and $\mathrm{Zn}$, the other metals showed low concentrations. Data comparison with Canadian standards for sediment quality (VGQS) showed Zn may pose a risk of biota contamination whereas $\mathrm{Cu}, \mathrm{Cd}, \mathrm{Pb}$ and $\mathrm{Cr}$ do not. In contrast to other metals which showed no significant differences between upstream and downstream, Zn showed higher concentration in upstream samples. In conclusion, the studied metals are associated with geological processes so the dairy activities do not contribute to stream contamination with these metals.

Keywords: Metals. Contamination. Dairy activities. 